\title{
Efficient Prediction of the First Just Noticeable Difference Point for JPEG Compressed Images
}

\section{Boban Bondžulić ${ }^{1}$, Nenad Stojanović1, Vladimir Petrović Boban Pavlović ${ }^{1}$, Zoran Miličević ${ }^{1}$}

${ }^{1}$ Military Academy, University of Defence in Belgrade

Veljka Lukića Kurjaka 33, 11000 Belgrade, Serbia

${ }^{2}$ Faculty of Technical Sciences, University of Novi Sad

Trg Dositeja Obradovića 6, 21000 Novi Sad, Serbia

e-mails: boban.bondzulic@va.mod.gov.rs,nenad.m.stojanovic@vs.rs, vladimir.petrovic@uns.ac.rs, boban.pavlovic@va.mod.gov.rs, zoran.milicevic@vs.rs

\footnotetext{
Abstract: In this paper, we show that a high-level of correlation exists between a simple image feature - mean gradient magnitude and the peak signal-to-noise ratio (PSNR) of the first just noticeable difference point for JPEG image compression. On the basis of this observation, we proposed a method to estimate the JPEG quality factor which represents the effective limit between perceptually lossy and lossless coding as the PSNR of the first just noticeable difference point. The goal is optimal image/video coding, at the lowest compression bit-rate that ensures perceptually lossless output image quality. We also show that this feature can be used to predict higher PSNR just noticeable difference points.
}

Keywords: JPEG; just noticeable difference points; peak signal-to-noise ratio; perceptually lossless

\section{Introduction}

In recent years there has been a rapid development of systems for digital processing, transmission, and display of image/video content [1, 2]. This development has led to great interest in efficient image compression techniques, which are capable of improving the compression ratio and image quality [3, 4].

Various compression standards have been developed for image archiving and transmission, such as JPEG and JPEG 2000, and intraframe profiles of H.264, H.265, and VVC video coding techniques have been used [5]. Among these techniques, JPEG compression is the most common due to its low complexity [6]. 
Image compression techniques eliminate coding and spatial redundancy, taking into account some of the features of the human visual system (HVS) - visual redundancy. Thanks to these features, various perceptual quantization schemes, and perceptual models have been developed and built into coding systems. Thus, by applying just noticeable difference (JND) models, compression efficiency can be improved. JND models estimate the maximum degradation of the visual signal that the HVS will not notice [7]. Therefore, JND can be viewed as a perceptual threshold in image/video processing applications and can be used for perceptual image compression. In addition to compression, JND is successfully used in the objective assessment of image quality, in all three assessment techniques (no-reference [8], reduced-reference [9], and full-reference [10]), leading to performance improvements.

Existing research on JND can be divided into three main areas: 1) subjective research with the aim of collecting JND annotations, 2) mathematical modeling of the distribution of JND points and 3) prediction of the distribution of JND points for a given image or video [5]. The research in this paper can be classified in the third area, with the aim of efficiently predicting the first JND point of images with JPEG compression.

\section{Related Works}

Traditional JND models can be divided into pixel-wise models, in which the JND threshold is determined for each pixel individually, and sub-band models in which JND thresholds are determined for each sub-band coefficient after switching to one of the transform domains. In most pixel-wise models, the effects of luminance adaptation and contrast masking are used, while in the sub-band model, the contrast sensitivity function plays a dominant role in determining the visibility threshold [11]. As in these models, the JND threshold is determined for each pixel or sub-band separately, and which does not properly reflect the total effect of image masking, in recent years picture-wise JND has been investigated, which measures the maximum image difference that HVS will not notice [12].

Recent research shows that observers differentiate between a finite number of image quality levels, as well as, that the relationship between perceptual distortions and bit-rate/distortion level is not a smooth but rather a step-wise function [13-15]. The steps of this function represent the JND points. The first and most significant JND point refers to the transition between a pristine and an image with visible distortions, or rather the transition from perceptually lossless to perceptually lossy encoding [16]. The second JND point was obtained by detecting noticeable differences from the first JND point (anchor). Lower JND points are used as anchors to determine higher JND points. 
JND points can be used to determine the satisfied-user-ratio (SUR) curves, taking into account that the user is satisfied with the visual test signal in relation to the reference visual signal, i.e. the difference in the quality of these two signals is below the JND threshold. SUR modeling that reflects user satisfaction is more suitable for streaming and coding applications than mean opinion scores which generally use a five-level scale (from the worst to excellent) [17].

JND-based subjective quality analysis has been conducted on JPEG [13, 18, 19], JPEG 2000 [19, 20], H.265 [20] and VVC compressed images [21, 22], and on H.264 [14, 15] and H.265 compressed videos [23], with results in publicly available JND-based image/video datasets.

Several JND prediction methods were proposed based on these data. JND prediction described in [24] uses local quality and masking models to form a feature vector used in a support vector regressor (SVR) with the aim of predicting the SUR curve from which the first JND can be derived. This method was extended to predict the second and third JND points in [25]. In [23] a feature set derived from the non-compressed source is input into a SVR to predict the first JND. A deep learning approach in [26] defines the quality factor of the first JND based on learning the SUR curve of the JPEG compressed image. In further research, the authors from [26] optimized the proposed architecture and applied a feature learning instead of a fine-tuning approach, which led to a significant reduction in computational cost and performance improvement [27]. A slidingwindow-based search strategy to predict JND based on a deep learning perceptually lossy/lossless predictor was proposed in [28]. These prediction methods can be used in perceptual quality assessment and adaptive perceptual image/video coding [29].

Research in this paper is focused on the most widespread type of image distortion, JPEG compression. Therefore, the JND analysis was performed on MCL-JCI image dataset [13], which contains information on the JND points of JPEG compressed images. This database was used to predict JND points in [26-28]. The mean absolute error (MAE) of the PSNR between the predicted and ground truth JND distributions was used as a prediction accuracy measure. In [26] a convolutional neural network using a 12,288-dimensional vector trained on 45 and tested on the remaining 5 sources over 10 iterations yielded a MAE for the first JND point of $0.69 \mathrm{~dB}$. The deep learning approach from [27] uses a 30,144dimensional vector, and it achieved an even better result in estimating the first JND point with an MAE of $0.58 \mathrm{~dB}$. Through five-fold cross-validation, the deep learning approach from [28] reached an MAE of $0.79 \mathrm{~dB}$.

In this paper, we show that a much simpler approach using the mean gradient magnitude (MGM) of the source non-compressed image can be used to reliably predict the first JND point of JPEG compression. The method does not require complex vision or masking models and determines the optimal JPEG quality factor (QF) through a simple rate-distortion function using the computationally efficient PSNR metric for objective quality assessment. Reaching the desired 
PSNR value of JPEG lossy compressed images has been the subject of various studies [30-34], and the results can be used here to accelerate the proposed approach.

\section{Dataset Description}

Prediction of the first JND (JND \#1) was performed on the MCL-JCI dataset, which consists of 5,000 JPEG compressed high-resolution images (1080x1920) [13], obtained by varying the JPEG quality factor from 1 (worst) to 100 (best quality) to generate 100 compressed images for each of the 50 different source images. A total of 150 observers evaluated the images using the bisection method with at least 30 scores gathered for each image. In this method, observers compare compressed images obtained with different quality factors and determine the visual threshold when there are no differences between them. Bisection search was adopted to speed up the procedure, i.e. to reduce the number of comparisons through an iterative procedure with division into half-quality intervals. The distribution of multiple JND points was modeled by a Gaussian mixture model [13].

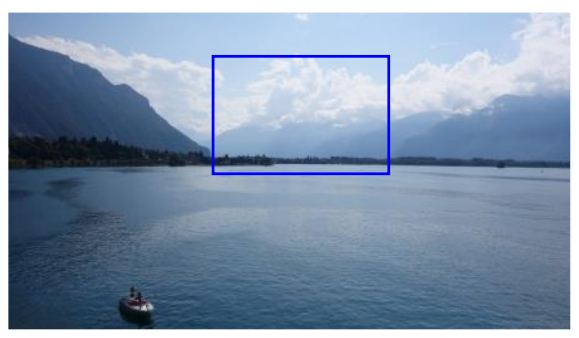

(a)

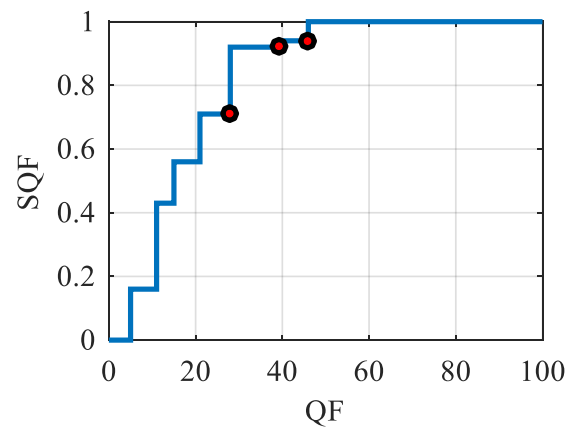

(c)

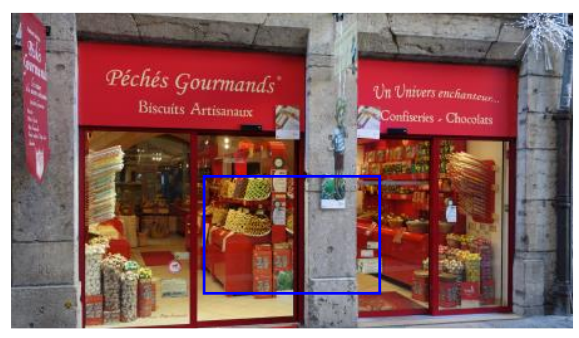

(b)

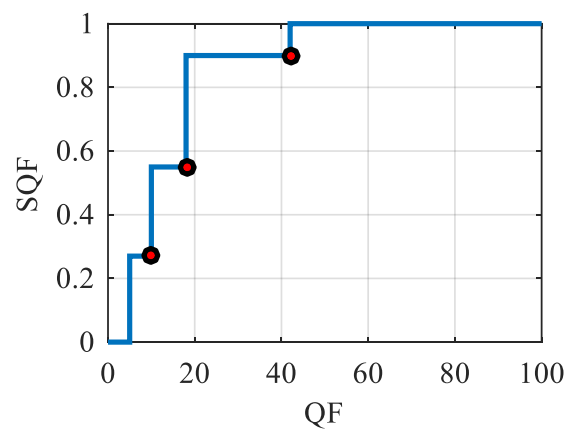

(d)

Figure 1

(a) original image with some large homogeneous areas, (b) original image rich with details, (c) and (d) are SQF functions of corresponding images above 


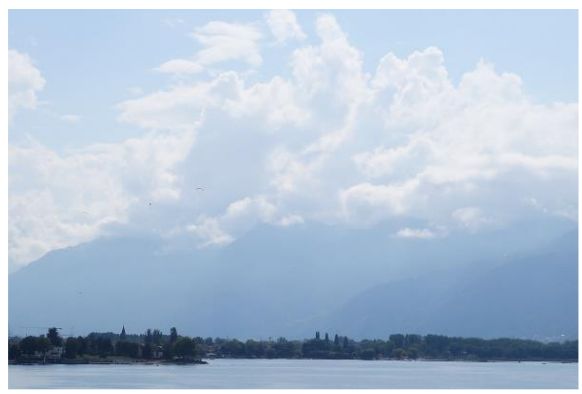

(a) original

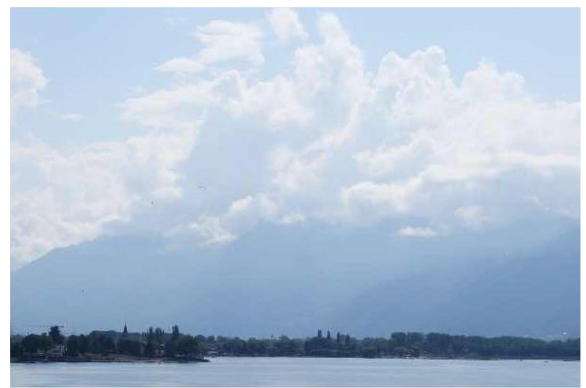

(c) JND \#1 (QF=46, SQF=0.94)

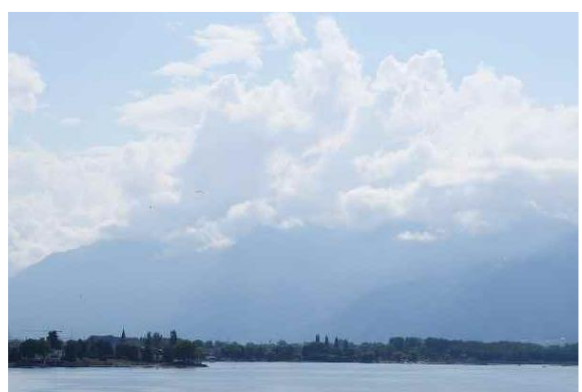

(e) JND \#2 (QF=39, SQF=0.92)

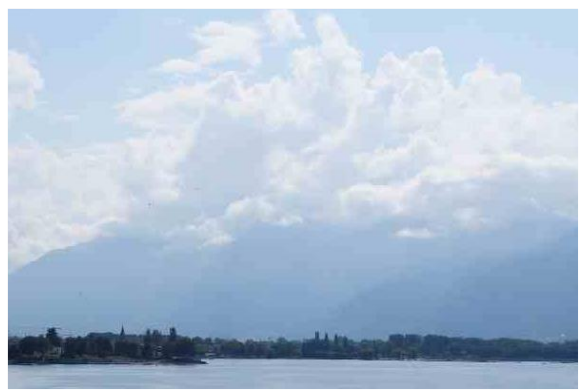

(g) JND \#3 (QF=28, SQF=0.71)

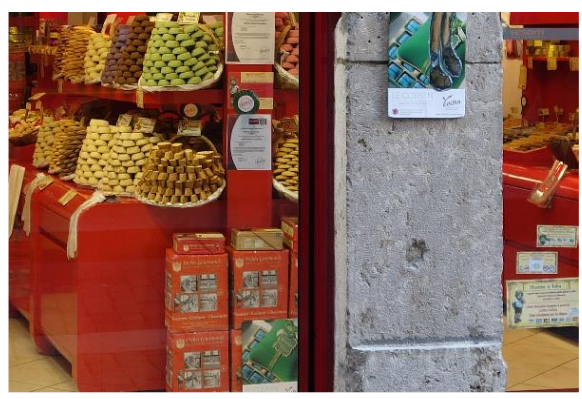

(b) original

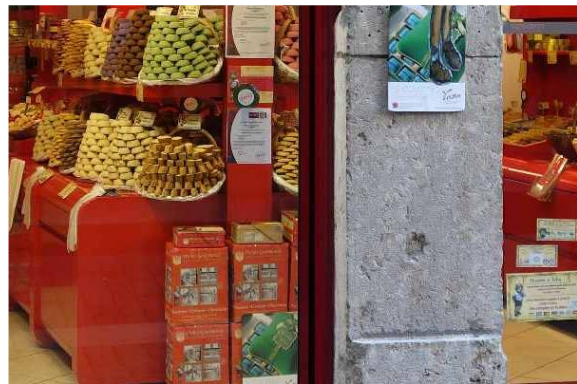

(d) JND \#1 (QF=42, SQF=0.90)

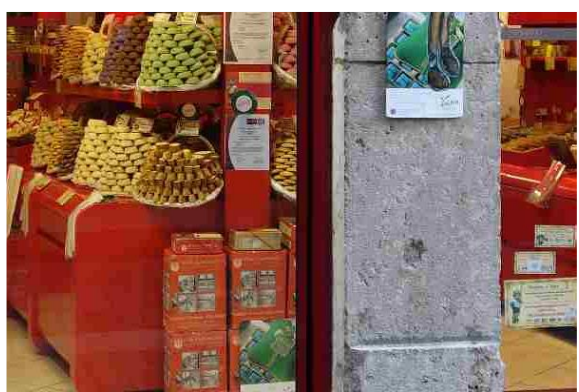

(f) JND \#2 (QF=18, SQF=0.55)

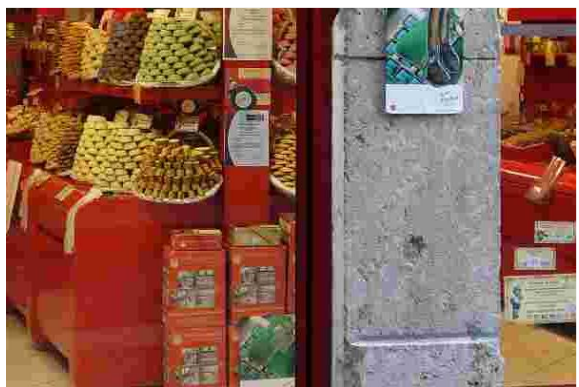

(h) JND \#3 (QF=10, SQF=0.27)

Figure 2

Regions from the original and JPEG coded images (denoted by the red dots in Fig. 1) 
The subjective trial results are presented through stair quality functions (SQF) obtained through analysis and post-processing of raw JND data. They show that human observers differentiate between 4 and 8 quality levels. Also, the results are mapped into $243 \mathrm{SQF}$ points (linking all quality factors which do not produce a noticeable difference), with each original image having at least 3 JND points mainly depending on the complexity of the depicted scene.

For images that are rich in detail, due to the masking effect, the number of JND levels (points) will be lower than for images that have large homogeneous regions. Examples for two images are given in Fig. 1, one with large homogeneous regions (Fig. 1a) and the other rich in detail (Fig. 1b). Along with original images, SQF functions (higher is better) are provided - Figs. 1c and 1d. Zoomed versions (blue rectangles in Figs. 1a and 1b) of original and JPEG coded images at three lowest successive SQF levels (red dots in Figs. 1c and 1d) are shown in Fig. 2.

From Fig. 2 it can be seen that the images corresponding to JND \#1 are of high quality and that there is no noticeable difference from the original. On the other hand, block effects can be seen in images corresponding to higher JNDs, and the difference from the original is noticeable.

\section{Results and Analysis}

As the images from MCL-JCI dataset are in RGB color format, their conversion to grayscale (luminance) images was first performed, using the weighted sum of the R (red), G (green) and B (blue) components:

$$
f(n, m)=0.299 R(n, m)+0.587 G(n, m)+0.114 B(n, m) .
$$

We analysed a set of simple features derived from the grayscale images in the MCL-JCI dataset - signal standard deviation, entropy, and MGM, and determined that MGM has the best agreement with PSNR of the first JND point. Specifically, MGM was determined on an $N x M$ grayscale image from the responses to $2 \mathrm{D}$ Sobel filters applied to it $\left(g_{x}\right.$ and $\left.g_{y}\right)$ :

$$
\begin{gathered}
g_{x}(n, m)=f(n+1, m-1)+2 f(n+1, m)+f(n+1, m+1), \\
-[f(n-1, m-1)+2 f(n-1, m)+f(n-1, m+1)]
\end{gathered},
$$

and

$$
\begin{gathered}
g_{y}(n, m)=f(n-1, m+1)+2 f(n, m+1)+f(n+1, m+1) \\
-[f(n-1, m-1)+2 f(n, m-1)+f(n+1, m-1)]
\end{gathered}
$$

From the resulting $g_{x}$ and $g_{y}$ oriented gradient components, MGM information is easily obtained according to: 
$M G M=\frac{1}{N M} \sum_{\forall n, m} \frac{1}{g_{\max }} \sqrt{g_{x}^{2}(n, m)+g_{y}^{2}(n, m)}$,

where $g_{\max }$ is the maximum magnitude value, taken as $g_{\max }=4.472$ for grayscale images with a dynamic range 0 to 1 [35] (image $\mathbf{f}$ which is an 8-bit unsigned integer array with a range of 0 to 255 is linearly scaled to a dynamic range of 0 to 1 with double-precision 64-bit format).

Mean gradient magnitude can be viewed as a descriptor of image contrast and texture, which are relevant for visibility masking estimation because in regions that contain more non-uniform contents more distortion can be tolerated than in regions with homogeneous content. This is confirmed in the Fig. 3, which shows the original uncompressed images from the MCL-JCI dataset with the smallest and largest MGM values. For these two images, the first JND points correspond to JPEG compression quality factors of $\mathrm{QF}=63$ and $\mathrm{QF}=32$ [13], respectively. Fig. 3 also shows JPEG compressed versions of the original images with $\mathrm{QF}=32$, which corresponds to the first JND point of the image with the maximum MGM value. For this image, no compression degradations are observed, while for an image with a minimum MGM value, the image degradations are noticeable in uniform regions.

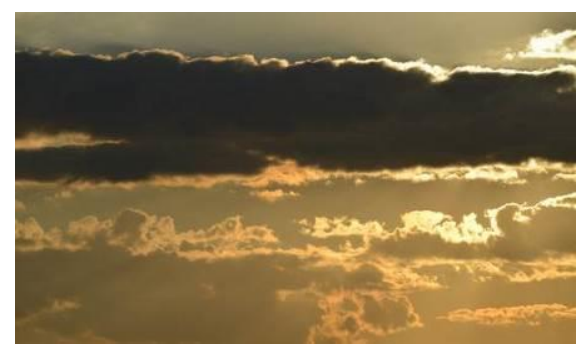

(a) original image $(\mathrm{MGM}=0.0122)$

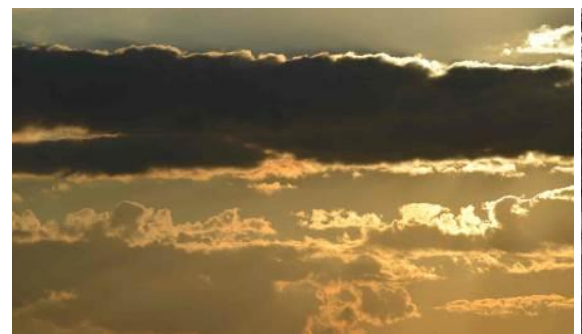

(c) JPEG compressed image $(\mathrm{QF}=32)$

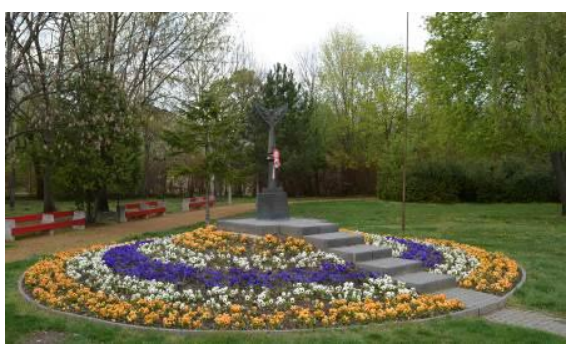

(b) original image $(\mathrm{MGM}=0.0902)$

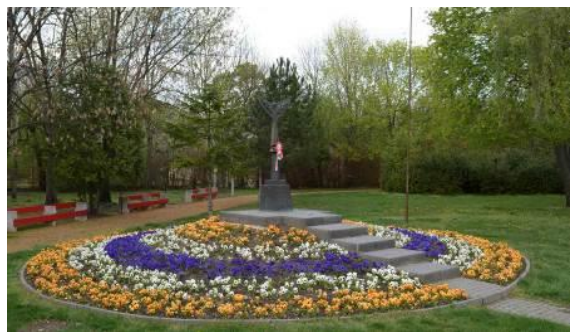

(d) JPEG compressed image $(\mathrm{QF}=32)$

Figure 3

Original images from the MCL-JCI dataset with: (a) the smallest and (b) the largest MGM values and (c) and (d) their JPEG compressed versions with $\mathrm{QF}=32$ 
PSNR is the most widely used full-reference metric and is defined via the mean squared error (MSE), computed by averaging the squared intensity differences of distorted (d) and reference (f) images, [36]:

$$
\begin{aligned}
& \operatorname{MSE}(\mathbf{d}, \mathbf{f})=\frac{1}{N M} \sum_{n=1}^{N} \sum_{m=1}^{M}(d(n, m)-f(n, m))^{2} \\
& \operatorname{PSNR}(\mathbf{d}, \mathbf{f})=10 \cdot \log _{10}\left(L^{2} / \operatorname{MSE}(\mathbf{d}, \mathbf{f})\right)
\end{aligned}
$$

where $N$ and $M$ are the image dimensions and $L$ is the dynamic range of the image.

Fig. 4 shows the correlation which we obtained between MGM and PSNR of JND \#1 modelled through a second-order mapping function. Based on this observation, we divided the MCL-JCI data into a training set of 25 randomly selected source images which we used to train a mapping function between MGM and corresponding PSNR of JND \#1 points on the condition that it is a falling function, which effectively stays constant after reaching its minimum:

$$
P S N R(M G M)=\left\{\begin{array}{c}
p_{1} M G M^{2}+p_{2} M G M+p_{3}, M G M \leq M G M_{c} \\
P S N R_{\text {min }}, M G M>M G M_{c}
\end{array},\right.
$$

where $M G M_{c}$ is the mean gradient magnitude for which the mapping function reaches its minimum value $\left(P S N R_{\min }\right)$.

Obtained least-squares fit parameters, $p_{1}, p_{2}$, and $p_{3}$ were then used to predict the PSNR of the JND \#1 of the remaining 25 source images (test set). Such randomised dataset division, training, and evaluation was repeated 200 times.

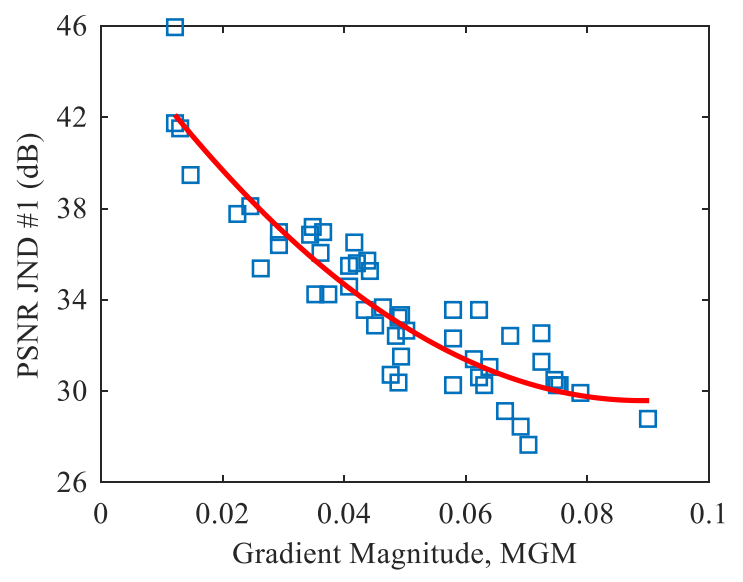

Figure 4

Mean gradient magnitude and PSNR of the first JND of the MCL-JCI source images (second order mapping function, solid line) 
Prediction function obtained using mean parameter values over the 200 training runs, $\left[p_{1}, p_{2}, p_{3}\right]=[2115.5,-377,46.4]$, is shown on Fig. 5 , and is given by:

$\operatorname{PSNR}(M G M)=\left\{\begin{array}{c}2115.5 M G M^{2}-377 M G M+46.4, M G M \leq 0.0896 \\ 29.58, M G M>0.0896\end{array}\right.$,

where for $M G M=0.0896$ the mapping function reaches its minimum value $\left(P S N R_{\min }=29.58 \mathrm{~dB}\right)$.

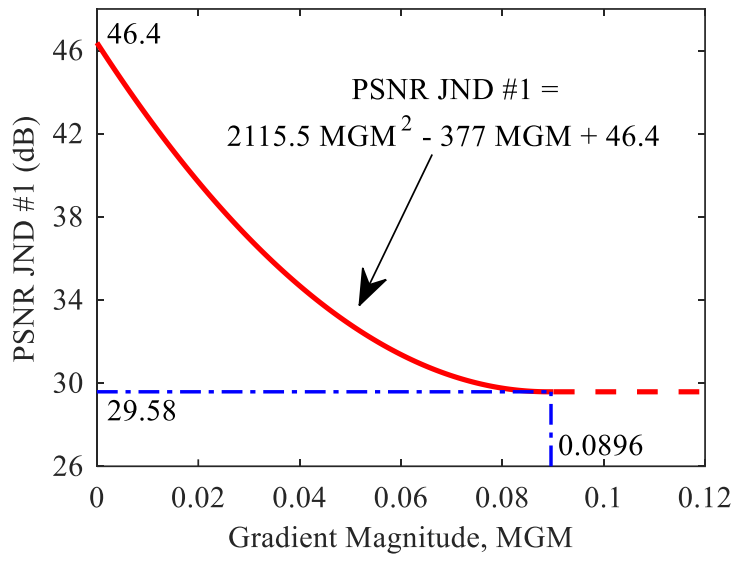

Figure 5

PSNR prediction of the first JND point based on MGM

Agreement between predicted and actual PSNR values of JND \#1 over 200 runs measured through linear correlation (LCC), Spearman and Kendall rank-order correlations (SROCC, KROCC), and MAE is provided in Table 1 (mean agreement). The metrics show a high level of agreement between the predicted and ground truth PSNR of JND \#1 (determined from subjective experiments).

Table 1

Mean agreement between the predicted and ground truth PSNR of JND \#1

\begin{tabular}{llll}
\hline LCC $(\%)$ & SROCC $(\%)$ & KROCC $(\%)$ & MAE $(\mathrm{dB})$ \\
\hline 91.54 & 90.44 & 75.05 & 1.21 \\
\hline
\end{tabular}

This can be compared to five- and ten-fold cross-validation deep learning approaches [26-28] which yielded a MAE of $0.69 \mathrm{~dB}, 0.58 \mathrm{~dB}$, and $0.79 \mathrm{~dB}$, respectively. We show that an MAE of just $0.6 \mathrm{~dB}$ more can be obtained with a significantly simpler and faster approach on a far less favourable $50 / 50 \%$ dataset split.

Fig. 6 shows predicted PSNR values of JND \#1, PSNR(MGM), obtained using the proposed approach and their relationship to the ground truth of the first three JND points, obtained through subjective trials and sorted in ascending JND \#1 order. 
Prediction function used mean parameter values obtained over the 200 training runs, Eq. 8.

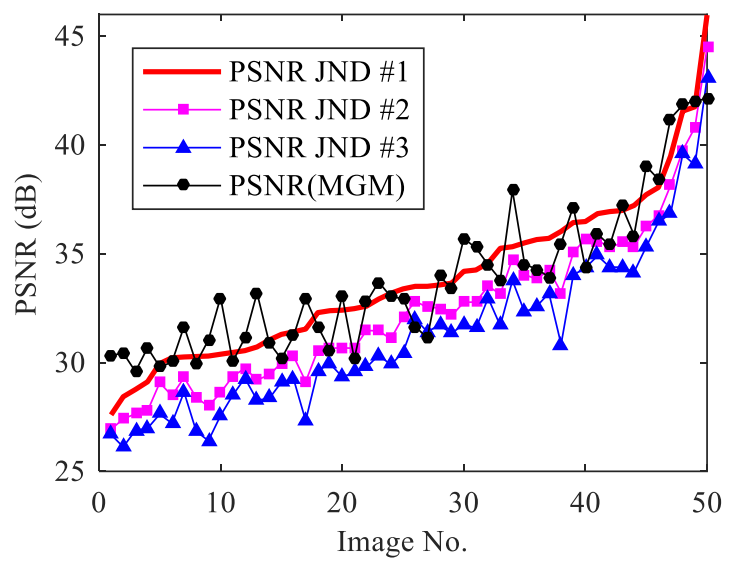

Figure 6

Predicted PSNR of JND \#1 and PSNR values of the first three JND points obtained from subjective trials

From Fig. 6 it can be seen that the predicted JND \#1 PSNR is greater than PSNR of JND \#2 in 43 out of 50 sources and greater than PSNR of JND \#3 in 46 out of 50 , which suggests that a small bias in the predicted PSNR, of $\sim 1.2 \mathrm{~dB}$ (MAE in Table 1), would eliminate practically all prediction errors and ensure that we always obtain an image with no visible compression artifacts. Another way to achieve this would be to use a minimum regional MGM instead of a globally determined value, which could focus on the most sensitive region but would also reduce the achievable compression ratios and thus our method's efficiency.

The proposed prediction method trained and validated on the MCL-JCI data was tested using independent high resolution (1600x1280) source images from a different dataset - JPEG XR [37]. Fig. 7 shows the relationship between PSNR and quality factor QF for JPEG XR images woman and $p 30$ with marked target JND \#1 PSNR and corresponding QF, 38 and 28 respectively. QF is shown in its full range, 1 to 100, while in real applications PSNR of JND \#1 could be sought in iterative procedures over a significantly narrower practical range. 


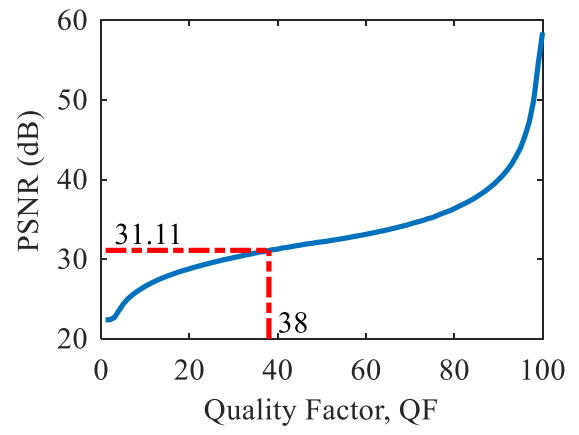

(a)

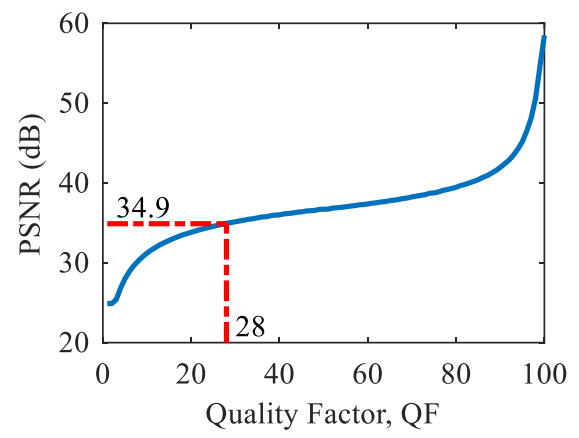

(b)

Figure 7

Relationship between PSNR and compression level (quality factor) of test images from the JPEG XR dataset: (a) test image woman and (b) test image $p 30$

Fig. 8 shows JPEG XR test images woman and $p 30$ optimally compressed using QF to match the PSNR of JND \#1 predicted through Eq. 8 using parameters defined above, where no distortions are apparent on normal viewing, as well as zoomed $160 \times 160$ pixel sections (1.25\% of image size) showing that images were indeed compressed with blocky artifacts visible only after enlargement - Fig. 8c and Fig. 8d. Using perceptually lossless compression preserves the quality of source signals while significantly reducing the bit length required to encode them from $2 \mathrm{MB}$ to 182 and $102 \mathrm{kB}$, with compression ratios of 11 and 20 respectively. For reference, lossless JPEG encoding on these images achieves image sizes of 1.36 $\mathrm{MB}$ and 1.17 MB respectively. Also, according to some research, websites such as Google and YouTube use JPEG compression with a QF of about 75 [5]. By applying the proposed method, significant bit-saving performance in signal archiving can be achieved, without perceptual loss of information (for the two selected images the optimal QF values are 38 and 28).

The results shown here were obtained for grayscale images, but analogous results can be achieved when the proposed method is applied to color images in the MCL-JCI dataset. In that case, even greater compression ratios can be achieved for pristine images.

Furthermore, we also found that a good correlation exists between MGM and PSNR of the higher JND points - Fig. 9, and we can use this to allow even greater latitude in practical systems setting quality/compression compromise automatically. Fig. 9d shows second-order PSNR prediction functions for the first four JND points, exhibiting a similar trend, with relative differences of approximately $1.4 \mathrm{~dB}$ (\#1 vs. \#2), $1.07 \mathrm{~dB}$ (\#2 vs. \#3) and $0.93 \mathrm{~dB}$ (\#3 vs. \#4). Linear correlation between the proposed second-order gradient-based prediction and ground truth PSNR of JND points \#2, \#3, and \#4 is above $91 \%$. 


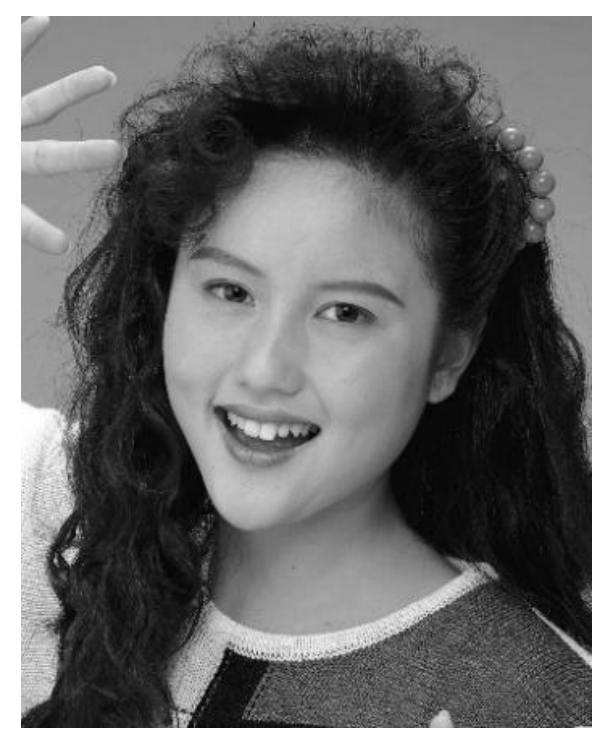

(a)

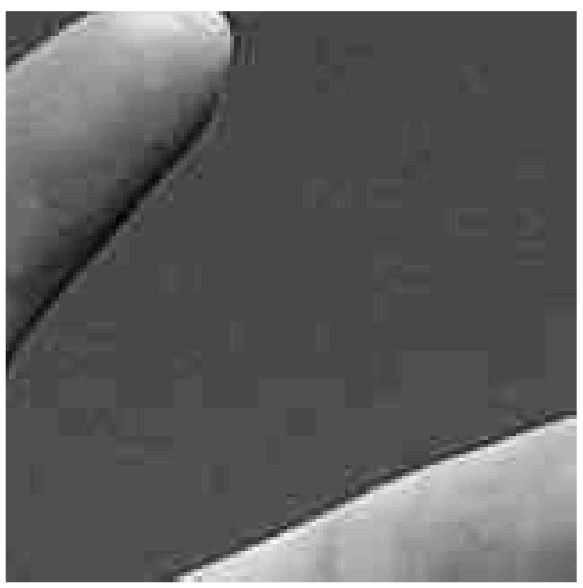

(c)

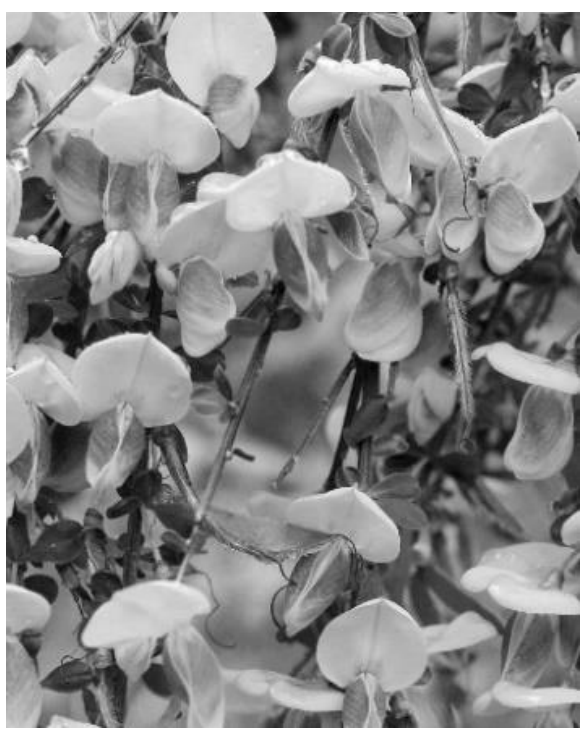

(b)

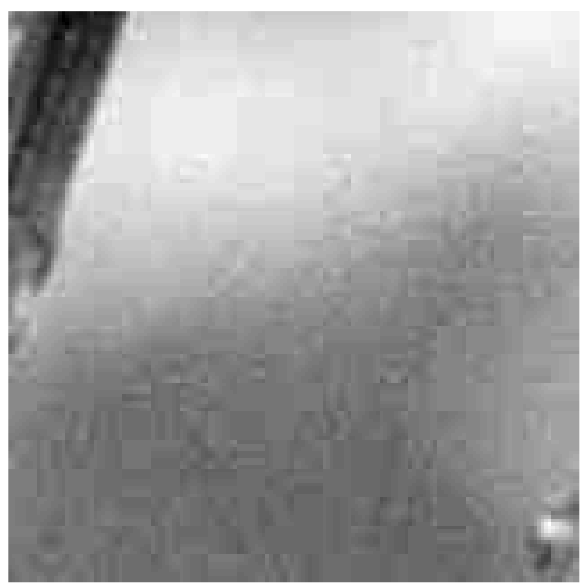

(d)

Figure 8

Test images compressed using the quality factor determined by the proposed method: (a) woman image compressed using JPEG QF=38, (b) p30 image compressed using JPEG QF=28, (c) top left 160x160 pixels of image on Fig. 8a and (d) central 160x160 pixels of image on Fig. 8b 


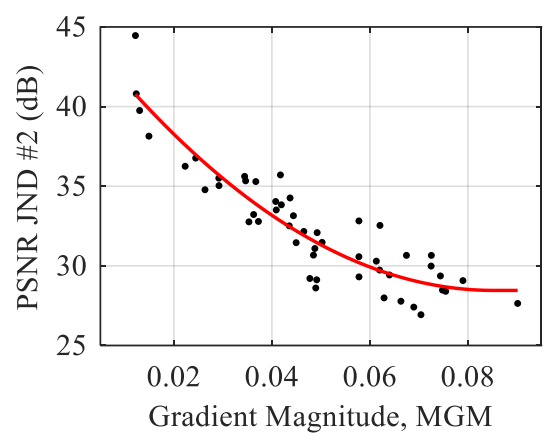

(a)

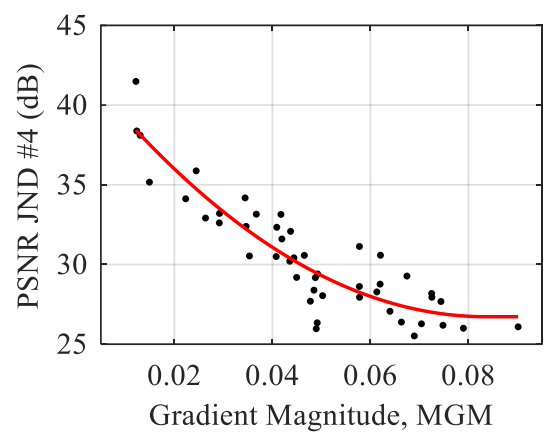

(c)

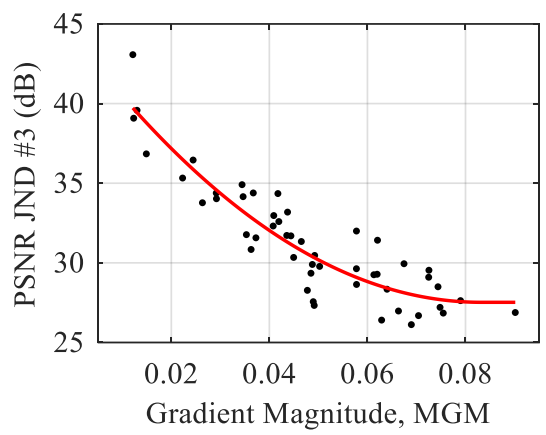

(b)

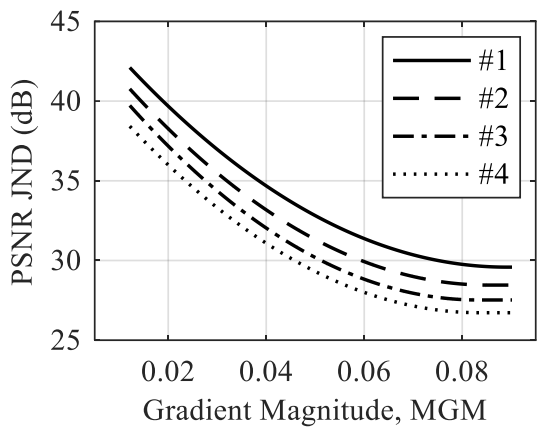

(d)

Figure 9

Predicted PSNR of JND: (a) \#2 (LCC=91.87\%), (b) \#3 (LCC=91.41\%), (c) \#4 (LCC=93.2\%) and (d) second order mapping functions for the first four JND points

One way to reach the desired PSNR value is by applying an iterative procedure with multiple compression and decompression, quality assessment, and changing the QF value [30]. However, thanks to the orthogonality of DCT, it is possible to estimate the PSNR of the spatial domain based on the MSE calculated in the DCT domain $[31,32]$. This avoids multiple compression/decompression.

Suppose that for an $p$-th block $(p=1, \ldots, P$, where $P$ is the total number of nonoverlapping blocks) reference image has a set of DCT coefficients $F(p, k, l)$ and the distorted image has a set of DCT coefficients $D(p, k, l)$, where $k=0, \ldots, 7$ and $l=0, \ldots, 7 . F(p, 0,0)$ and $D(p, 0,0)$ are the direct current (DC) coefficients that relate to the means in the $p$-th blocks. The differences between coefficients in the block are:

$\Delta D(p, k, l)=D(p, k, l)-F(p, k, l)$,

and based on them the MSE in the $p$-th block can be determined:

$$
M S E_{p}=\frac{1}{64} \sum_{k=0}^{7} \sum_{l=0}^{7}(\Delta D(p, k, l))^{2} .
$$


Finally, the estimates of MSE and PSNR in the DCT domain for the entire image are:

$$
\begin{aligned}
& M S E_{D C T}=\frac{1}{P} \sum_{p=1}^{P} M S E_{p}=\frac{1}{64 P} \sum_{p=1}^{P} \sum_{k=0}^{7} \sum_{l=0}^{7}(\Delta D(p, k, l))^{2}, \\
& P S N R_{D C T}=10 \cdot \log _{10}\left(L^{2} / M S E_{D C T}\right) .
\end{aligned}
$$

Fig. 10 shows a scatter plot between the PSNR values calculated in spatial (Eq. 6) and in DCT domains (Eq. 12) for 243 JPEG images from MCL-JCI dataset (all JND points). It is easy to see that the PSNR values calculated in the spatial domain are practically equal to the PSNR values calculated in the DCT domain.

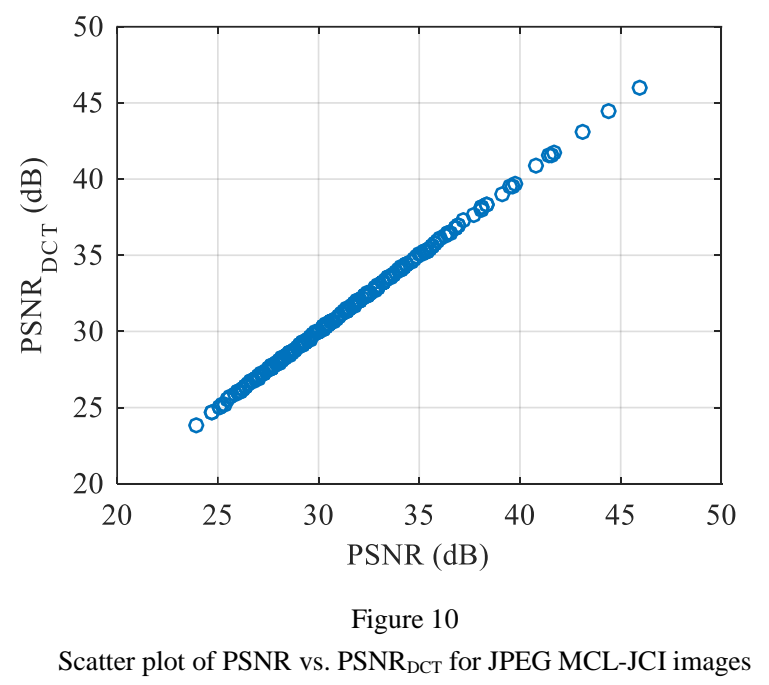

The relationship between the PSNR values calculated in the spatial and DCT domains is further illustrated in Fig. 11, using a different degree of compression of the same image (test image $p 30$ from the JPEG XR dataset). In the DCT domain, compression was introduced using quality factors, on the basis of which quantization matrices are determined [4]. In this case, too, it is noticed that the PSNR values calculated in the spatial domain are practically the same as the PSNR values calculated in the DCT domain. In this way, by determining the array of DCT coefficients of all image blocks, it is possible to make a prediction of MSE and PSNR. Additionally, by changing the quality factor QF, it is possible to adjust the QF to reach the desired MSE or PSNR value without iterative compression/decompression. Although in this way an accelerated process of reaching the desired value of PSNR has been achieved, in our future research we will analyze the achievement of the desired value of PSNR JND \#1 without iterations [33], through two steps [34] and through the use of a limited number of blocks [30]. 


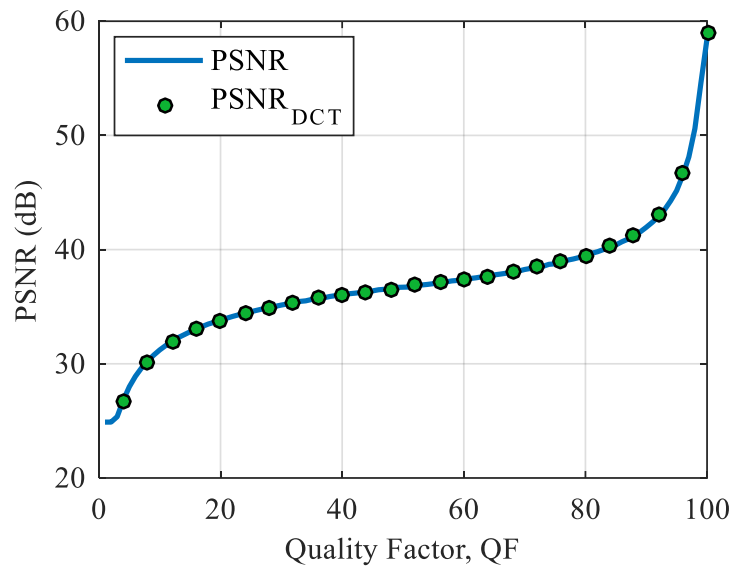

Figure 11

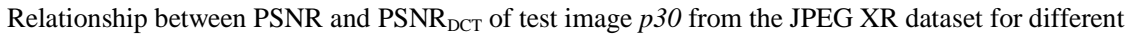
compression levels (quality factors)

Prediction of the first JND point for MCL-JCI JPEG compressed images can be achieved in the PSNR, QF, and bits per pixel (bpp) domains [28]. Therefore, the correlation between ground truth values (determined from subjective experiments) and their predictions in these three domains were analyzed (Eq. 8 is used for prediction with optimal set parameters $\left[p_{1}, p_{2}, p_{3}\right]$ defined above). The scatter plots between predicted and ground truth measures values of JND \#1 are shown in Fig. 12, and quantitative indicators of the degree of their agreement (correlations) are given in Table 2 .

Fig. 12 and Table 2 show the lowest degree of agreement between QF ground truth and predicted values $(\mathrm{LCC}=40.58 \%)$. In the vicinity of the $\mathrm{QF}$ ground-truth value of JND \#1 there is a very wide region of QF values for which the difference between compressed images is very difficult to observe by observers (see Fig. 1). The width of this region depends on the content of the image. The wider this region, the higher the probability that the predicted values will deviate from the ground truth values, so there is a small degree of their mutual correlation. The degree of agreement between PSNR ground truth and predicted values are significantly higher ( $\mathrm{LCC}=92.02 \%$ ), so it can be concluded that it is necessary to use the PSNR domain to predict the first JND point.

Table 2

Agreement between the predicted and ground truth PSNR, bpp and QF of JND \#1

\begin{tabular}{llll}
\hline & LCC $(\%)$ & SROCC $(\%)$ & KROCC $(\%)$ \\
\hline PSNR & 92.02 & 91.38 & 75.18 \\
\hline bpp & 78.71 & 80.04 & 61.96 \\
\hline QF & 40.58 & 48.37 & 35.37 \\
\hline
\end{tabular}




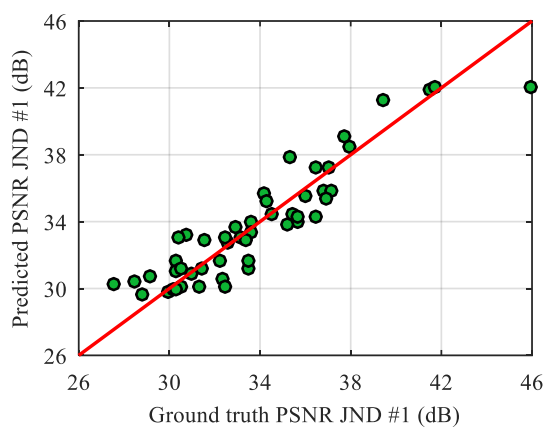

(a)

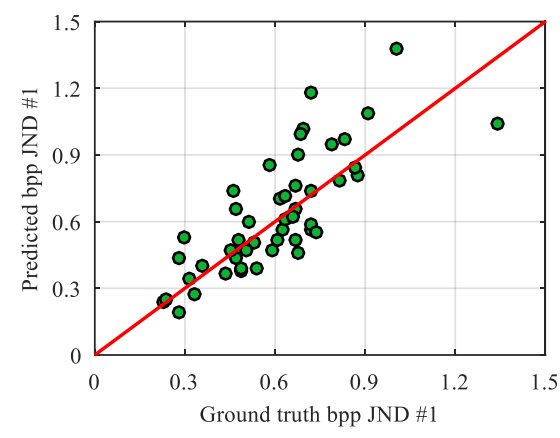

(b)

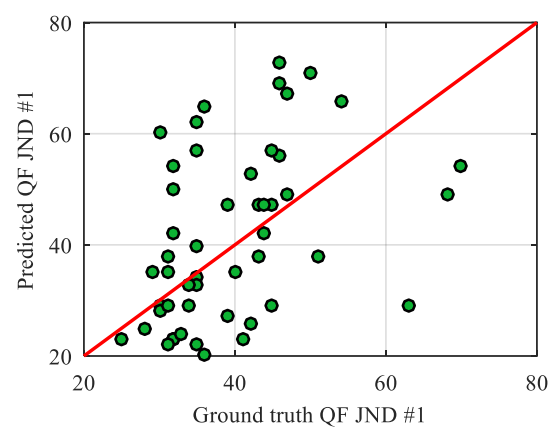

(c)

Figure 12

Predicted vs. ground truth metrics of JND \#1: (a) PSNR, (b) bpp and (c) QF

\section{Conclusions}

This paper demonstrated that simple to compute mean gradient magnitude can be used to reliably predict the PSNR of the first just noticeable difference point as the limit between perceptually lossless and lossy JPEG image coding. This allows adaptive setting and optimisation of compression ratios meaning the proposed method can efficiently reach optimally high compression ratios without visible distortions or information loss. Furthermore, it can be used to set optimal compression ratios over a wider range of increasingly perceptually visible differences points. Additionally, this approach can be used to guide watermark or hidden message embedding, allowing the embedded information to remain undetected by observers. The agreement (linear correlation) between the proposed second-order gradient-based prediction and ground truth PSNR for the first four JND points is above $91 \%$. Furthermore, the mean absolute error between predicted and ground-truth PSNR values of the first and the most important just noticeable difference points is $1.21 \mathrm{~dB}$, which is only $0.6 \mathrm{~dB}$ worse than the best result achieved by applying a significantly more complex deep learning approach on the same image dataset. Also, the paper shows that reaching the desired value of 
PSNR can be accelerated by its determination in the DCT domain. The proposed picture-wise approach can be used in the efficient compression of grayscale and color images.

As different parts of the image (or blocks) may have non-uniform and homogeneous content, different just noticeable difference values correspond to them. Therefore, one of the directions of further research will be a block-based prediction of perceptual visual redundancy. Also, in further work, the possibility of applying the gradient magnitude as a feature in the video compression just noticeable difference points will be analyzed.

\section{Acknowledgment}

This research has been a part of the project No. VA-TT/3/20-22 supported by the Ministry of Defence, Republic of Serbia.

\section{References}

[1] Bovik, A. C.: Automatic prediction of perceptual image and video quality, Proceedings of the IEEE, 2013, Vol. 101, No. 9, pp. 2008-2024

[2] Ilic, S., Petrovic, M., Jaksic, B., Spalevic, P., Lazic, Lj., Milosevic, M.: Experimental analysis of picture quality after compression by different methods, Przeglad Elektrotechniczny, 2013, Vol. 89, No. 11, pp. 190-194

[3] Ernawan, F., Kabir, N., Zamli, K. Z.: An efficient image compression technique using Tchebichef bit allocation, Optik - International Journal for Light and Electron Optics, 2017, Vol. 148, pp. 106-119

[4] Poth, M., Trpovski, Z., Loncar-Turukalo, T.: Analysis and improvement of JPEG compression performance using custom quantization and block boundary classifications, Acta Polytechnica Hungarica, 2020, Vol. 17, No. 6, pp. 171-191

[5] Tian, T., Wang, H., Zuo, L., Kuo, C.-C. J., Kwong, S.: Just noticeable difference level prediction for perceptual image compression, IEEE Transactions on Broadcasting, 2020, Vol. 66, No. 3, pp. 690-700

[6] Wang, Z., Tran, T.-H., Muthappa, P. K., Simon, S.: A JND-based pixeldomain algorithm and hardware architecture for perceptual image coding, Journal of Imaging, 2019, Vol. 5: 50, pp. 1-29

[7] Wu, J., Shi, G., Lin, W.: Survey of visual just noticeable difference estimation, Frontiers of Computer Science, 2019, Vol. 13, pp. 4-15

[8] Kim, M., Song, K.-S., Kang, M.-G.: No-reference image contrast assessment based on just-noticeable-difference, in Proc. IS\&T International Symposium on Electronic Imaging - Image Quality and System Performance XIV, Burlingame, CA, USA, 2017, pp. 26-29 
[9] Miao, X., Lee, D.-J.: Just-noticeable difference binary pattern for reduced reference image quality assessment, Optical Engineering, 2019, Vol. 58, No. 9, Article no. 093105

[10] Seo, S., Ki, S., Kim, M.: A novel just-noticeable-difference-based saliencychannnel attention residual network for full-reference image quality predictions, IEEE Transactions on Circuits and Systems for Video Technology, 2021, Vol. 31, No. 7, pp. 2602-2616

[11] Wang, H., Yu, L., Liang, J., Yin, H., Li, T., Wang, S.: Hierarchical predictive coding-based JND estimation for image compression, IEEE Transactions on Image Processing, 2021, Vol. 30, pp. 487-500

[12] Li, H., Jenadeleh, M., Chen, G., Reips, U.-D., Hamzaoui, R., Saupe, D.: Subjective assessment of global picture-wise just noticeable difference, in Proc. IEEE International Conference on Multimedia \& Expo Workshops, London, UK, 2020, pp. 1-6

[13] Jin, L., Lin, J. Y., Hu, S., Wang, H., Wang, P., Katsavounidis, I., Aaron, A., Kuo, C.-C. J.: Statistical study on perceived JPEG image quality via MCLJCI dataset construction and analysis, in Proc. IS\&T International Symposium on Electronic Imaging - Image Quality and System Performance XIII, San Francisco, CA, USA, 2016, Article no. IQSP-222

[14] Wang, H., Gan, W., Hu, S., Lin, J. Y., Jin, L., Song, L., Wang, P., Katsavounidis, I., Aaron, A., Kuo, C.-C. J.: MCL-JCV: a JND-based H.264/AVC video quality assessment dataset, in Proc. IEEE International Conference on Image Processing, Phoenix, AZ, USA, 2016, pp. 1509-1513

[15] Wang, H., Katsavounidis, I., Zhou, J., Park, J., Lei, S., Zhou, X., Pun, M.O., Jin, X., Wang, R., Wang, X., Zhang, Y., Huang, J., Kwong, S., Kuo, C.C. J.: VideoSet: a large-scale compressed video quality dataset based on JND measurement, Journal of Visual Communication and Image Representation, 2017, Vol. 46, pp. 292-302

[16] Huang, J., Feng, H., Xu, Z., Li, Q., Chen, Y.: A robust deblurring algorithm for noisy images with just noticeable blur, Optik - International Journal for Light and Electron Optics, 2018, Vol. 168, pp. 577-589

[17] Zhang, X., Yang, C., Wang, H., Xu, W., Kuo, C.-C. J.: Satisfied-user-ratio modeling for compressed video, IEEE Transactions on Image Processing, 2020, Vol. 29, pp. 3777-3789

[18] Liu, X., Chen, Z., Wang, X., Jiang, J., Kwong, S.: JND-Pano: database for just noticeable difference of JPEG compressed panoramic images, Lecture Notes in Computer Science, 2018, Vol. 11164, pp. 458-468

[19] Ahar, A., Mahmaoudpour, S., Van Wallendael, G., Paridaens, T., Lambert, P., Schelkens, P.: A just noticeable difference subjective test for high dynamic range images, in Proc. $10^{\text {th }}$ International Conference on Quality of Multimedia Experience, Cagliari, Italy, 2018, pp. 1-6 
[20] Fan, C., Zhang, Y., Hamzaoui, R., Jiang, Q.: Interactive subjective study on picture-level just noticeable difference of compressed stereoscopic images, in Proc. IEEE International Conference on Acoustics, Speech and Signal Processing, Brighton, UK, 2019, pp. 8548-8552

[21] Shen, X., Ni, Z., Yang, W., Zhang, X., Wang, S., Kwong, S.: A JND dataset based on VVC compressed images, in Proc. IEEE International Conference on Multimedia \& Expo Workshop, London, United Kingdom, 2020, pp. 1-6

[22] Shen, X., Ni, Z., Yang, W., Zhang, X., Wang, S., Kwong, S.: Just noticeable distortion profile inference: a patch-level structural visibility learning approach, IEEE Transactions on Image Processing, 2021, Vol. 30, pp. 26-38

[23] Huang, Q., Wang, H., Lim, S. C., Kim, H. Y., Jeong, S. Y., Kuo, C.-C. J.: Measure and prediction of HEVC perceptually lossy/lossless boundary QP values, in Proc. Data Compression Conference, Snowbird, UT, USA, 2017, pp. $42-51$

[24] Wang, H., Katsavounidis, I., Huang, Q., Zhou, X., Kuo, C.-C. J.: Prediction of satisfied user ratio for compressed video, in Proc. IEEE International Conference on Acoustics, Speech and Signal Processing, Calgary, Canada, 2018, pp. 6747-6751

[25] Wang, H., Zhang, X., Yang, C., Kuo, C.-C. J.: Analysis and prediction of JND-based video quality model, in Proc. Picture Coding Symposium, San Francisco, CA, USA, 2018, pp. 278-282

[26] Fan, C., Lin, H., Hosu, V., Zhang, Y., Jiang, Q., Hamzaoui, R., Saupe, D.: SUR-Net: predicting the satisfied user ratio curve for image compression with deep learning, in Proc. $11^{\text {th }}$ International Conference on Quality of Multimedia Experience, Berlin, Germany, 2019, pp. 1-6

[27] Lin, H., Hosu, V., Fan, C., Zhang, Y., Mu, Y., Hamzaoui, R., Saupe, D.: SUR-FeatNet: Predicting the satisfied user ratio curve for image compression with deep feature learning, Quality and User Experience, 2020, Vol. 5, No. 5, pp. 1-23

[28] Liu, H., Zhang, Y., Zhang, H., Fan, C., Kwong, S., Kuo C.-C. J., Fan, X.: Deep learning based picture-wise just noticeable prediction model for image compression, IEEE Transactions on Image Processing, 2020, Vol. 29, pp. 641-656

[29] Yuan, D., Zhao, T., Xu, Y., Xue, H., Lin, L.: Visual JND: a perceptual measurement in video coding, IEEE Access, 2019, Vol. 7, pp. 29014-29022

[30] Kozhemiakin, R., Lukin, V., Vozel, B.: Image quality prediction for DCTbased compression, in Proc. $14^{\text {th }}$ International Conference The Experience of Designing and Application of CAD Systems in Microelectronics, Lviv, Ukraine, 2017, pp. 225-228 
[31] Kozhemiakin, R. A., Abramov, S. K., Lukin, V. V., Vozel, B., Chehdi, K.: Output MSE and PSNR prediction in DCT-based lossy compression of remote sensing images, in Proc. Image and Signal Processing XXIII, Warsaw, Poland, 2017, Article no. 1042721

[32] Lu, K.-S., Ortega, A., Mukherjee, D., Chen, Y.: Perceptually inspired weighted MSE optimization using irregularity-aware graph Fourier transform, in Proc. IEEE International Conference on Image Processing, Abu Dhabi, United Arab Emirates, 2020, pp. 3384-3388

[33] Minguillon, J., Pujol, J.: JPEG standard uniform quantization error modeling with applications to sequential and progressive operation modes, Journal of Electronic Imaging, 2001, Vol. 10, No. 2, pp. 475-485

[34] Krivenko, S., Demchenko, D., Dyogtev, I., Lukin, V.: A two-step approach to providing a desired quality of lossy compressed images, in Proc. Nechyporuk, M., Pavlikov, V., Kritskiy D. (eds): Integrated Computer Technologies in Mechanical Engineering, Advances in Intelligent Systems and Computing, 2020, Vol. 1113, pp. 482-491

[35] Bondzulic, B., Petrovic, V., Andric, M., Pavlovic, B.: Gradient-based image quality assessment, Acta Polytechnica Hungarica, 2018, Vol. 15, No. 4, pp. 83-99

[36] Bondzulic, B. P., Pavlovic, B. Z., Petrovic, V. S., Andric, M. S.: Performance of peak signal-to-noise ratio quality assessment in video streaming with packet losses, Electronics Letters, 2016, Vol. 52, pp. 454456

[37] De Simone, F., Goldmann, L., Baroncini, V., Ebrahimi, T.: Subjective evaluation of JPEG XR image compression, in Proc. SPIE 7443, San Diego, CA, USA, 2009, Article no. 74430L 